\title{
New options to fight Ebola
}

The latest Ebolavirus (EBOV) outbreak in West Africa remains uncontrolled, partially because there are still no approved vaccines or therapeutics to prevent or treat these infections. Over the past few decades, several EBOV vaccine candidates have advanced to clinical trials, all of which are based on their ability to elicit immune responses against EBOV glycoproteins (GPs; the major viral immunogens): DNA-based vaccines; replication-incompetent chimpanzee recombinant adenoviruses (rAds); and live-attenuated recombinant vesicular stomatitis viruses (rVSVs).

Now, three studies present the preliminary results of Phase I clinical trials for rAd- and rVSV-based vaccine candidates. Zhu et al. recruited 120 participants in China who were either treated with placebo or a single low dose $\left(4 \times 10^{10}\right.$ viral particles $)$ or a single high-dose $\left(16 \times 10^{10}\right.$ viral particles) rAd-based vaccine expressing the GP of the 2014 epidemic Makona EBOV strain. The vaccine seemed to be safe and generated a dose-dependent antibody and cellular immune response against GP. Agnandji et al. tested an rVSVbased vaccine expressing the GP of the Mayinga EBOV strain (rVSVZEBOV) in a total of 158 participants in four trials in Africa and Europe. They tested vaccine doses ranging from 300,000 plaque-forming units $(\mathrm{PFU})$ to $50 \times 10^{6} \mathrm{PFU}$ and found no serious vaccine-related adverse effects, although vaccine-induced viraemia was detected in almost all participants receiving more than $3 \times 10^{6} \mathrm{PFU}$, and up to one-fifth of the participants developed viral-induced arthritis. All doses of the vaccine were found to induce GP-specific antibodies, with higher vaccine doses correlating with higher titres of neutralizing antibodies. Similarly, Regules et al. tested the rVSV-ZEBOV vaccine at a low dose $\left(3 \times 10^{6} \mathrm{PFU}\right)$ and a high dose $\left(20 \times 10^{6} \mathrm{PFU}\right)$ in two different locations in the United States. The vaccine induced a dosedependent antibody response against GP and seemed to be safe, although the authors also observed transient vaccine-induced viraemia but did not report any cases of vaccine-induced arthritis. Collectively, these studies show that these vaccine candidates are promising and could be moved into Phase II clinical trials.

Furthermore, two new studies characterized novel vaccines that are protective against lethal EBOV challenge in nonhuman primates. Mire et al. addressed the safety concerns related to the vaccine-induced viraemia that characterizes the rVSV-ZEBOV vaccine by generating two new attenuated rVSV-based strains (rVSVN1CT1GP3 and rVSVN4CT1GP1). Notably, compared with rVSV-ZEBOV, a single dose $\left(2 \times 10^{7} \mathrm{PFU}\right)$ of these new vaccines resulted in reduced levels of vaccineinduced viraemia in cynomolgus macaques. Both attenuated strains elicited antibodies against GP and protected macaques from infection with a lethal dose (1,000 PFU) of the Makona EBOV strain. In a separate study, Marzi et al. developed a wholevirus vaccine based on a replicationdefective Mayinga strain of EBOV that lacks VP30 (EBOVDVP30), which is an essential viral transcription activator. Notably, a single dose ( $10^{7}$ focus-forming units (FFU)) of this whole-virus vaccine was sufficient to generate a strong antibody and cellular immune response in cynomolgus macaques and protected the animals from challenge with a lethal dose (1,000 FFU) of the heterologous Kikwit strain of EBOV. Owing to concerns about the use of a whole-virus EBOV vaccine, the authors chemically inactivated the EBOV $\triangle$ VP30 strain with hydrogen peroxide and found that it still induced a strong immune response and protected macaques from lethal EBOV challenge. Together, these two studies describe novel vaccine candidates that may be used in future clinical trials.

Finally, Thi et al. treated rhesus monkeys infected with a lethal dose (1,000 PFU) of the Makona EBOV strain with lipid nanoparticleencapsulated short interfering RNAs (siRNAs) that target multiple sequences of the viral genome. Treatment was started 3 days post infection and all treated animals survived, whereas untreated controls succumbed on day 8 or 9 post infection. Furthermore, siRNA treatment reduced the plasma viral load and provided moderate protection against liver dysregulation and renal dysfunction, suggesting that this therapeutic strategy may confer benefits other than increased survival and the control of viral replication.

Taken together, these studies expand the current arsenal of therapeutics that are available to prevent and treat Ebola infections, and some of these candidates are already being tested in the most affected areas of West Africa.

Cláudio Nunes-Alves

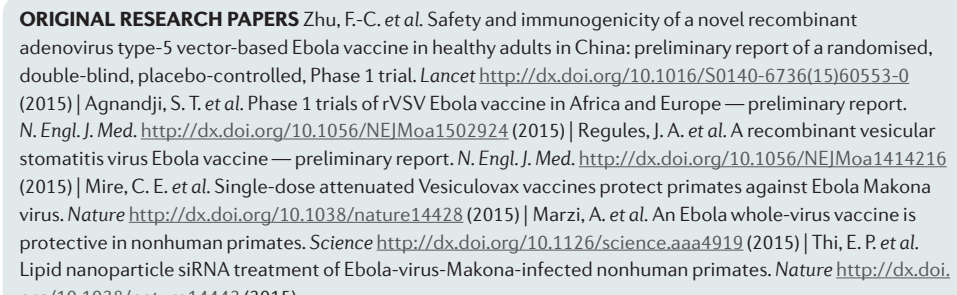

